\title{
Longitudinal Effects of Nortriptyline on EEG Sleep and the Likelihood of Recurrence in Elderly Depressed Patients
}

Daniel J. Buysse, M.D., Charles F. Reynolds, III, M.D., Carolyn C. Hoch, Ph.D., Patricia R. Houck, M.S.H., David J. Kupfer, M.D., Sati Mazumdar, Ph.D., and Ellen Frank, Ph.D.

Our objectives were to determine the effects of nortriptyline and placebo on subjective and EEG sleep measures over 1 year of maintenance therapy in elderly depressed patients and to determine the relationship of such effects to recurrence in nortriptyline or placebo-treated patients during maintenance therapy.

EEG and subjective sleep assessments were conducted before and during a maintenance therapy study of patients suffering from major depression. During acute treatment all patients received nortriptyline plus interpersonal psychotherapy (IPT). During maintenance treatment patients were randomly assigned to double-blind treatment in one of four cells: nortriptyline with IPT; nortriptyline with medication clinic (no IPT); placebo with IPT; or placebo with medication clinic. Sleep evaluations were conducted at one point before treatment, one point following remission during continuation nortriptyline/IPT treatment, and at three time points after random assignment to maintenance treatment.

The setting was the sleep laboratory of the outpatient depression treatment clinic, and subjects were a convenience sample of media-recruited and clinically referred elderly outpatient depressed patients $(\mathrm{n}=72)$.
Complete sleep analyses were conducted for 21 nortriptylineand 10 placebo-treated patients throughout 1 year of maintenance treatment. The main outcome measures were subjective and EEG sleep measures and the recurrence of major depression.

Our results show that nortriptyline acutely and persistently decreased REM sleep, increased phasic REM activity, decreased sleep apnea, and had no effect on periodic limb movements during sleep. Recurrence on maintenance nortriptyline was associated with lower phasic REM activity during early continuation therapy, but EEG sleep measures did not predict recurrence during placebo maintenance therapy. Patients treated with nortriptyline had a lower recurrence rate than those treated with placebo. Better subjective sleep quality and maintenance IPT were associated with a lower rate of recurrence regardless of nortriptyline treatment. It seems that nortriptyline has persistent effects on REM sleep and sleep apnea in elderly depressed patients. Maintenance nortriptyline, maintenance IPT, good subjective sleep quality, and high-phasic REM activity are associated with a reduced likelihood of the recurrence of depression during maintenance therapy. [Neuropsychopharmacology 14:243-252 1996]
From the Mental Health Clinical Research Center for the Study of Late-Life Mood Disorders, Department of Psychiatry, University of Pittsburgh School of Medicine, Pittsburgh, PA.

Address correspondence to Dr. Buysse, 3811 O'Hara Street, Pittsburgh, PA 15213.

Received October 31, 1994; revised May 30, 1995; accepted June 8, 1995.
Major depression is associated with consistent EEG sleep changes that are particularly evident among elderly patients (Reynolds and Kupfer 1987; Knowles and MacLean 1990; Benca et al. 1992). Sleep EEG features in depressed patients, such as the delta ratio (Kupfer et al. 1990) and REM latency (Giles et al. 1987), are also associated with the likelihood and timing of future depressive recurrences. Furthermore, the base- 
line measure of delta ratio interacts with continued psychotherapy during maintenance treatment to influence time to recurrence (Kupfer et al. 1990).

Antidepressant medications also have well-recognized effects on EEG sleep that "normalize" some of the typical depressive sleep changes (summarized in Buysse 1991). The most consistent antidepressant drug effect is REM sleep suppression, although the potency of this effect ranges from very strong, with agents such as clomipramine and monamine oxidase inhibitors (Dunleavy and Oswald 1973; Kupfer et al. 1989) to relatively minor, with agents such as trimipramine (Dunleavy et al. 1972; Ware et al. 1989). In general antidepressant drugs do not increase slow-wave sleep, but some drugs such as trazodone increase the total amount of stage 3 and 4 sleep (Montgomery et al. 1983; Mouret et al. 1988), and drugs such as nortriptyline and clomipramine increase the amount of delta EEG activity during the first NREM period relative to the entire night (Reynolds et al. 1991; Kupfer et al. 1993). Some studies have demonstrated that specific patterns of change in EEG sleep during early drug therapy also correlate with ultimate clinical response (Kupfer et al. 1981; Reynolds et al. 1991).

Although the acute effects of antidepressant drugs (up to 4 weeks) have been well documented, there have been very few studies of longer-term treatment. Given current recommendations for longer treatment durations in acute major depression (American Psychiatric Association 1993), understanding the longer-term effects of these medications on sleep could improve further our understanding of their neurobiology and mechanisms of action. Kupfer et al. (1994) recently reported on EEG sleep effects of imipramine over 3 years of maintenance therapy in middle-aged depressed patients. The study showed persistence of changes observed in the acute medication treatment phase throughout the maintenance interval. Furthermore, Kupfer and colleagues have also demonstrated that delta EEG counts during continuation therapy with imipramine were significantly associated with the likelihood of recurrence during a 3-year follow-up (Kupfer et al. 1993).

The current study also examines long-term effects of antidepressant medications on sleep, but we have focused on a group of elderly patients with recurrent major depression. The long-term sleep effects in this group may be particularly salient because of their high frequency of subjective sleep complaints on the one hand and concern about anticholinergic and cardiovascular effects of medications on the other hand. We examined EEG sleep during a 1-year maintenance pharmacotherapy with nortriptyline in elderly depressed patients, contrasting these results with a parallel group of patients randomized to maintenance placebo treatment for 1 year; within each group patients were further randomized for maintenance interpersonal psychotherapy (IPT). The specific goals of the current study were (1) to examine the effects of nortriptyline on EEG sleep over 12 months of maintenance therapy in elderly depressed patients; (2) to determine whether EEG sleep measures at baseline or during continuation nortriptyline therapy are associated with recurrence during a 1-year followup; and (3) to determine whether maintenance IPT further influences the likelihood of recurrence or continued wellness. Based on the results reported by Kupfer and colleagues, we predicted that acute nortripytline effects on sleep would persist during maintenance and that reduced delta EEG counts would predict recurrence in both placebo- and nortriptyline-treated groups. We also expected maintenance nortriptyline and maintenance interpersonal psychotherapy to increase the likelihood of continued wellness during the 1-year follow-up.

\section{METHODS}

\section{Study Description}

The current results are part of a larger study of EEG sleep and clinical outcome during maintenance pharmacotherapy and psychotherapy of late-life depression (Reynolds et al. 1991, 1992). Elderly patients with recurrent unipolar major depression first receive acute treatment with a combination of nortriptyline and IPT. Remission of the depressive episode is defined by three consecutive weeks of a Hamilton Depression Rating Scale (HRSD); (Hamilton 1960) score of $\leq 10$. The acute treatment phase typically lasts for 12 to 24 weeks. Once initial remission has been achieved, patients continue nortriptyline in the same dose for an additional 16 weeks, together with IPT every other week. At this point, a 6-week transition phase begins, wherein subjects are randomly assigned to one of four cells for maintenance therapy: nortriptyline plus IPT; nortriptyline plus "medication clinic"; placebo plus IPT; or placebo plus "medication clinic." For those assigned to placebo, nortriptyline is gradually tapered and discontinued over this 6-week period. Medication clinic consists of brief (15-minute) weekly meetings with a clinician and physician focusing on symptoms of depression and medication effects or side effects; no interpersonal or other specific psychotherapy issues are addressed. Patients in the four maintenance cells did not differ significantly on clinical characteristics including age, sex ratio, duration of depressive episode, number of previous episodes, or severity of the index episode. Following the transition phase a 3-year maintenance phase begins. All patients are followed monthly during maintenance therapy, with the goal of rapidly identifying recurrence of major depression. Recurrence is defined by syndromal major depression (RDC) with a HRSD score $\geq 17$ for 2 consecutive weeks.

The current study focused primarily on two groups 
of patients, placebo- and nortriptyline-treated patients, each treated over a period of 12 months. Within nortriptyline and placebo groups, we further identified two subgroups, those with a recurrence within 12 months, and those successfully completing 12 months of maintenance therapy. The nortriptyline group consists of 33 patients, including 24 who completed 12 months of follow-up and 9 who suffered a recurrence. Among the placebo-treated group, 14 completed 1 year of maintenance treatment without recurrence and 25 suffered a recurrence.

\section{Patients}

The study group included patients aged 60 to 80 years with a diagnosis of recurrent unipolar nonpsychotic major depression determined by the Schedule for Affective Disorders and Schizophrenia (Spitzer 1978). A severity rating of $\geq 17$ on the 17 -item HRSD is required for study entry. Furthermore, patients are required to have stable medical conditions posing no contraindication to nortiptyline, and not to be taking any medications that could directly cause depressive symptoms. A total of $46 / 72$ depressed patients in the final sample were taking medications at the baseline evaluation. These medications included nonsteroidal antiinflammatory drugs $(n=18)$, diuretics $(n=14)$, calcium-channel antagonists $(n=11)$, thyroid replacement hormones $(n=$ $8)$, digoxin $(n=5)$, and estrogen/progresterone $(n=5)$; fewer than five subjects were taking medications of other classes (e.g., vasodilators, hypoglycemic agents). Written informed consent was obtained for each subject before participation in the study.

To date, a total of 148 patients have been accepted into the study and 86 have been randomly assigned to maintenance treatment. The smaller number of patients reported in the current analysis (72) reflects the number of patients who had sleep studies at the relevant time points and for whom we could determine a one-year outcome (recurrence or completion). Some patients did not have baseline sleep studies because the acuteness of their depression required immediate intervention or because of medication factors that would preclude an adequate study. Other subjects have not yet completed 1 year of maintenance treatment. Figure 1 displays subject flow for patients included in this report.

\section{EEG Sleep Studies}

We report EEG sleep studies from five time points: (1) baseline while depressed and not on medication $\left(T_{1}\right)$; (2) after remission of clinical symptoms during nortriptyline continuation treatment $\left(T_{2}\right)$; (3) at 1 month into maintenance treatment after placebo-nortriptyline assignment $\left(T_{3}\right)$; and again (4) at $6\left(T_{4}\right)$ and (5) 12 months (T5) into the maintenance phase of treatment (Figure 1). Thus all patients in the current report were antidepressant-medication free at the initial time point, and all were taking nortriptyline during the second sleep evaluation. The mean nortripytline dose at $T_{2}$ was $85.2 \pm$ $36.9 \mathrm{mg}$ (range $20-200$ ), producing a mean plasma level of $90.5 \pm 24.0 \mathrm{ng} / \mathrm{ml}$ (range 34-149). The nortriptylineand placebo-assigned subjects diverged in medication status at the 1-month $\left(T_{3}\right), 6$-month $\left(T_{4}\right)$, and 12-month $\left(T_{5}\right)$ maintenance therapy time points. At each evaluation point, the patients completed three consecutive nights of EEG sleep studies. In addition to routine EEG, EOG, and EMG measurements, patients were monitored for sleep apnea and periodic limb movements (PLM) on the first night of each series. High- and lowfilter frequency settings for the EEG and EOG were 30 and $0.3 \mathrm{~Hz}$, with a sensitivity of $5 \mu \mathrm{V} / \mathrm{mm}$. All records were visually scored for traditional domains of sleep continuity, sleep architecture, and REM phasic and temporal measures. Each EEG record was also analyzed with period analysis for determination of $0.5-$ to $3.0-\mathrm{Hz}$ EEG delta activity during NREM sleep, and with an automated REM detection algorithm during REM epochs (Kupfer et al. 1984; Doman et al. 1995). These analyses exclude epochs identified as awake or movement time. In addition to EEG sleep measures, we also examined subjective sleep quality using the Pittsburgh Sleep Quality Index (PSQI; Buysse et al. 1989).

\section{Statistical Analyses}

We selected nine sleep measures a priori as outcome variables. These represent the domains of sleep continuity, sleep state architecture, phasic REM, and delta EEG activity and were selected because each has been found to characterize depressive sleep disturbance in previous investigations.

Descriptive statistics are presented as means with standard deviations or medians with ranges, as appropriate. Group comparisons were performed using $t$-tests. Repeated-measures analyses of variance (ANOVA) was used to contrast sleep variables in nortriptyline- and placebo-treated patients during the maintenance phase; this analysis used one between-group factor and one repeated measure with four time points (continuation treatment with nortriptyline, and 1-, 6-, and 12-month maintenance treatment after nortriptyline-placebo randomization). Exploratory analyses for placebo completers and recurrences used group $t$-tests. The effects of nortriptyline on sleep apnea and PLMs were assessed using the Wilcoxon sign-rank test, a nonparametric paired test of central tendency. Finally, we used Cox proportional hazards models to identify significant sleep and clinical covariates of time to recurrence during maintenance treatment. The Wald $\chi^{2}$ was used as a test of statistical significance in these analyses. 


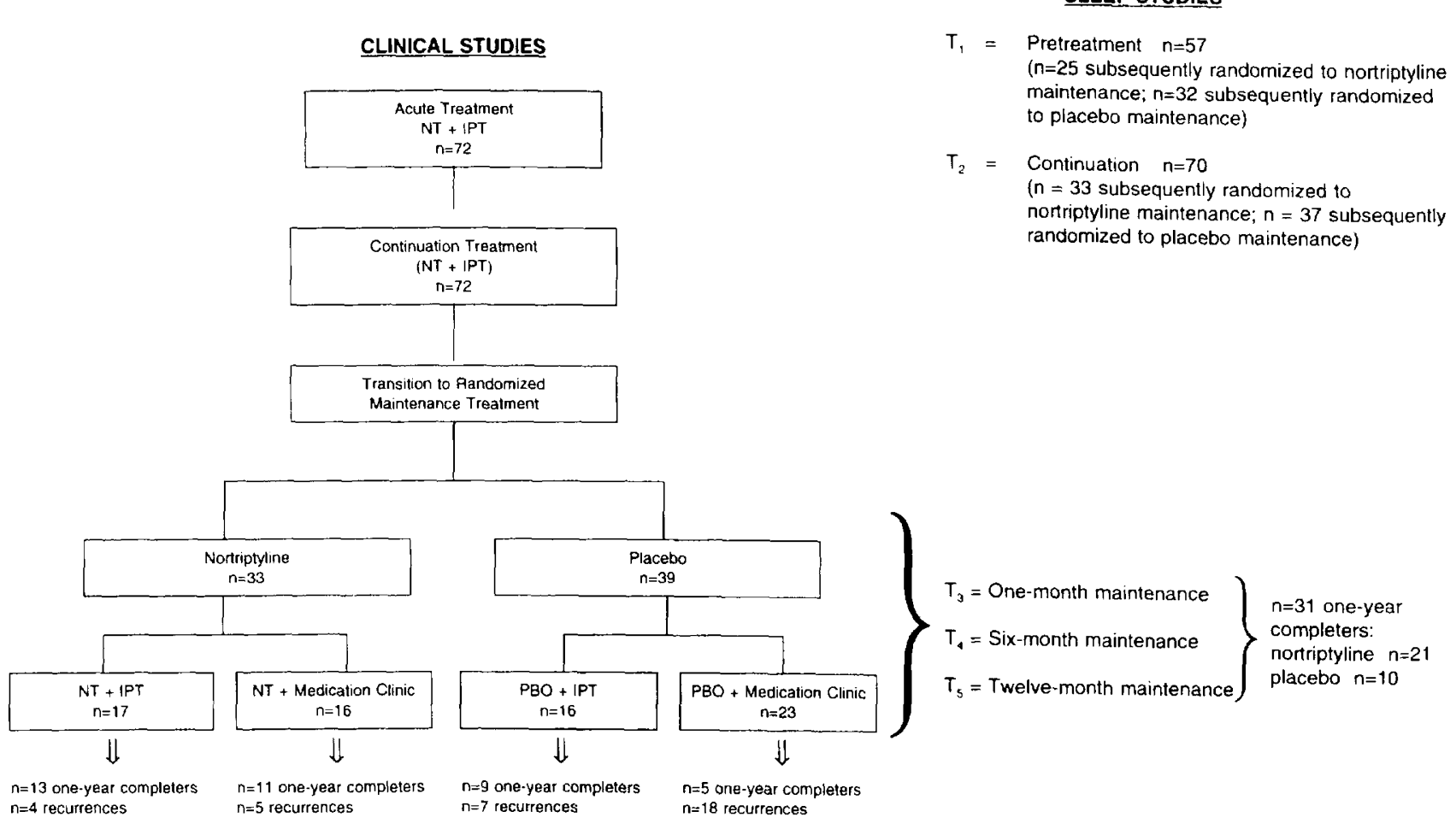

Figure 1. Study outline. Clinical phases and sleep evaluation points are shown in parallel.

\section{RESULTS}

\section{Effects of Nortriptyline versus Placebo during Maintenance Treatment}

For these analyses we contrasted the 21 patients who remained well for 1 year during nortriptyline maintenance therapy with the 10 patients who remained well during placebo maintenance therapy. (This represents the subgroup of patients who have completed sleep studies at each time point and who have remained well throughout the first year of maintenance tyerapy.) Results of these analyses are summarized in Table 1 and Figures 2-4.

Group Effects (Nortriptyline versus Placebo). Neither subjective sleep quality measured by the PSQI nor EEG sleep maintenance showed significant group differences over the 12 months of maintenance therapy. Sleep architecture showed a significant and persistent reduction in REM sleep percent among the nortriptylinetreated group $(p<.04)$. REM density was significantly elevated in this group $(p<.0001)$. The groups did not differ in total delta EEG counts, but the nortriptylinetreated group showed some evidence for increased delta counts early in the sleep period, as evidenced by a higher delta sleep ratio (average number of delta counts per minute in NREM 1 divided by average number of delta counts per minute in NREM 2; $p<.09$ ). REM latency did not differ between the two groups.
Time and Group $\times$ Time Interaction Effects. There was an overall trend toward decreasing PSQI scores, that is, improved sleep quality, over time. The group-by-time interaction was not significant, indicating no difference in the temporal trends of the nortriptyline and placebo groups. None of the sleep EEG measures showed any significant change over time. Furthermore, the nortriptyline- and placebo-treated patients did not have different time courses, as indicated by the absence of significant group-by-time interactions for any sleep EEG measure.

Apnea and Periodic Limb Movements. To examine sleep apnea and PLMs as a function of nortriptyline treatment, we compared subjects at $T_{1}$ and $T_{2}$. Of the total sample, 53 subjects had complete apnea and PLM data at $T_{1}$ and $T_{2}$. Among this group the apnea-hypopnea index decreased from a mean of $5.5 \pm 7.5$ events per hour at baseline $\left(T_{1}\right)$ to a mean of $3.3 \pm 5.0$ events per hour during continuation $\left(T_{2}\right)$ (Wilcoxon sign rank test, $p<.005)$; median values decreased from 2.3 to 1.4. The total number of NREM apneas decreased from a mean of $22.1 \pm 42.4$ to $13.8 \pm 24.0$ (Wilcoxon sign rank test, $p<.08$ ), but the mean number of events during REM sleep did not change significantly. In a similar way, the apnea-hypopnea index during NREM decreased significantly, from $5.6 \pm 8.9$ to $2.7 \pm 5.2$ (Wilcoxon sign rank test, $p<.05$ ), but the apnea-hypopnea index during REM did not change significantly $(8.8 \pm 14.1$ to $7.1 \pm$ 8.5). For 38 patients with complete oximetry data, the 


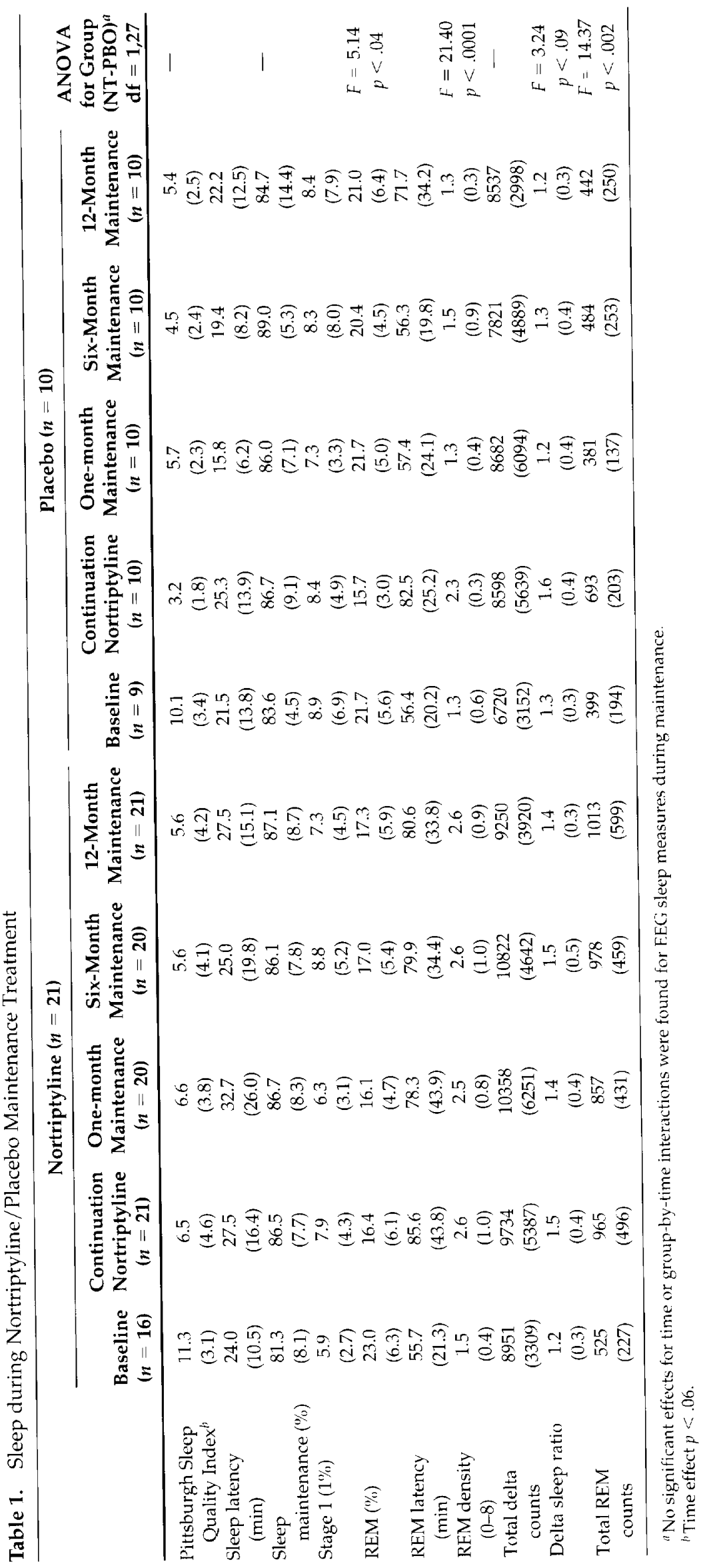




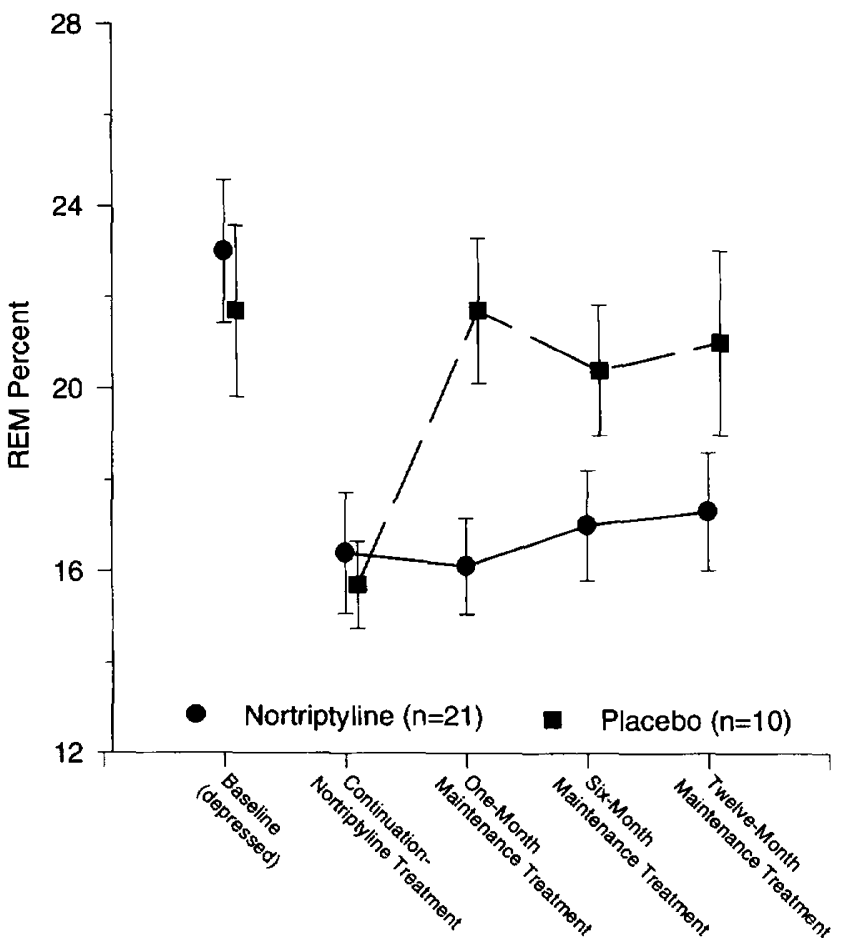

Figure 2. Nortriptyline suppresses REM sleep acutely and persistently. Nortriptyline and placebo-treated patients differed significantly $(F=5.14, p<.04)$. Data are shown as means with standard error bars. Nortriptyline $(n=21)$ placebo $(n=10)$

mean minimum saturation was $82.2 \pm 8.2$ at baseline and $81.6 \pm 7.5$ during continuation (NS). The PLM arousal index was $10.2 \pm 14.6$ at baseline and $10.5 \pm$ 13.1 during early nortriptyline continuation, and median PLM arousal indices were 4 and 5.1. These differences were not statistically significant.

As a further measure of nortriptyline effects on apnea and PLMs over time, we compared 15 nortriptyline patients with complete data at both continuation $\left(T_{2}\right)$ and 12-month maintenance $\left(T_{5}\right)$ with six placebotreated patients with complete data at the same time points. We found no significant difference between groups in the change of the apnea-hypopnea index, total events during NREM or REM sleep, or periodic limb movement arousal index.

\section{Clinical and Sleep EEG Correlates of Treatment Outcome}

Nortriptyline maintenance treatment was associated with a significantly lower recurrence rate compared to placebo $\left(27 \%\right.$ vs. $\left.62 \% ; \chi^{2}=8.56, p<.003\right)$. We examined whether baseline $\left(T_{1}\right)$ clinical or sleep EEG baseline measures significantly influenced time to recurrence using a Cox proportional hazards model stratifying on medicatíon group (nortriptyline vs. placebo). This analysis included 33 patients eventually randomized to nortrip-

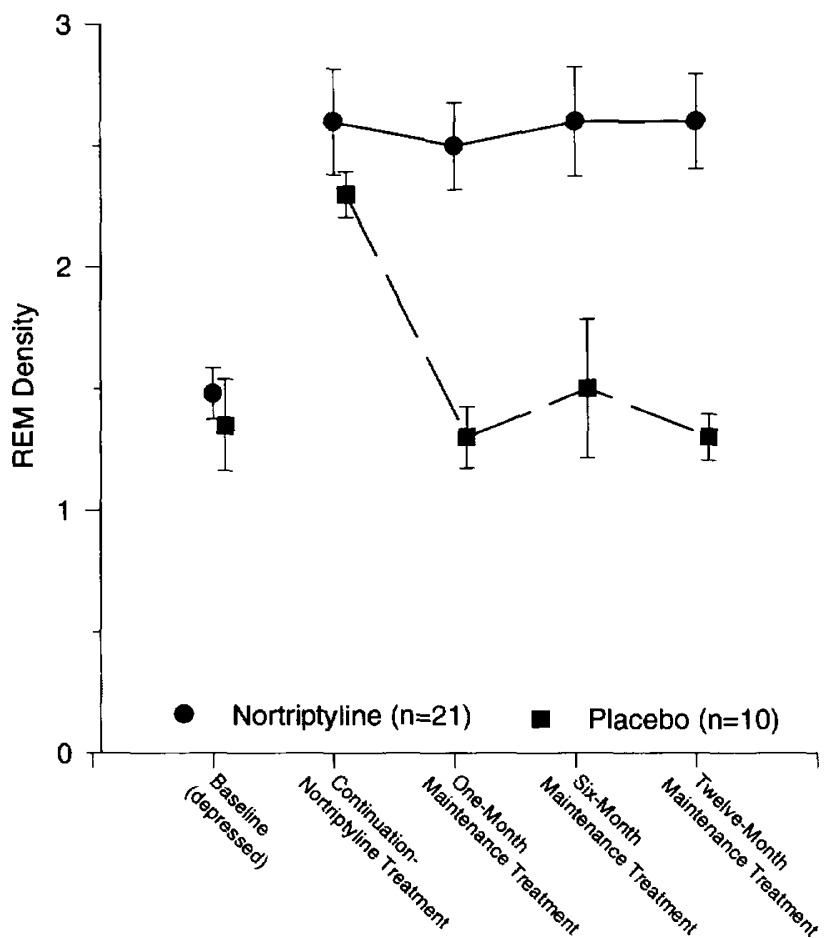

Figure 3. Nortriptyline increases REM density acutely and persistently. REM density is defined as the amount of visually estimated REM phasic activity divided by total REM sleep time. Nortriptyline and placebo-treated patients differed significantly $(F=21.40, p<.0001)$. - Nortriptyline $(\mathrm{n}=$ 21) Placebo $(\mathrm{n}=10)$

tyline and 39 patients eventually randomized to placebo maintenance. An initial analysis with clinical measures found no effect of sex, age, or educational status on treatment outcome. A separate analysis used baseline $\left(T_{1}\right)$ measures of sleep quality (PSQI global score), REM density, and REM latency as continuous covariates, as well as the presence or absence of IPT during maintenance therapy as a dichotomous covariate. This analysis included 25 patients subsequently randomized to nortriptyline, and 32 subsequently randomized to placebo. Again we found no significant correlates. Finally, we used the same four measures during early nortriptyline continuation therapy $\left(T_{2}\right)$. For this analysis complete data were available for 33 patients subsequently randomized to NT and 37 patients subsequently randomized to placebo maintenance. PSQI global score, REM density, and maintenance IPT were significant correlates of time to recurrence $\left(\chi^{2}=6.54, p<.02 ; \chi^{2}=4.29, p<.05 ;\right.$ and $\chi^{2}=$ $4.05, p<.05$, respectively). Patients with better subjective sleep quality and higher REM density at the continuation assessment $\left(T_{2}\right)$ and who received maintenance IPT were less likely to suffer recurrence during the first year of maintenance therapy. Risk ratios were 0.491 for IPT, 0.59 for REM density, and 1.150 for PSQI.

Acute changes in sleep with drug treatment may also 


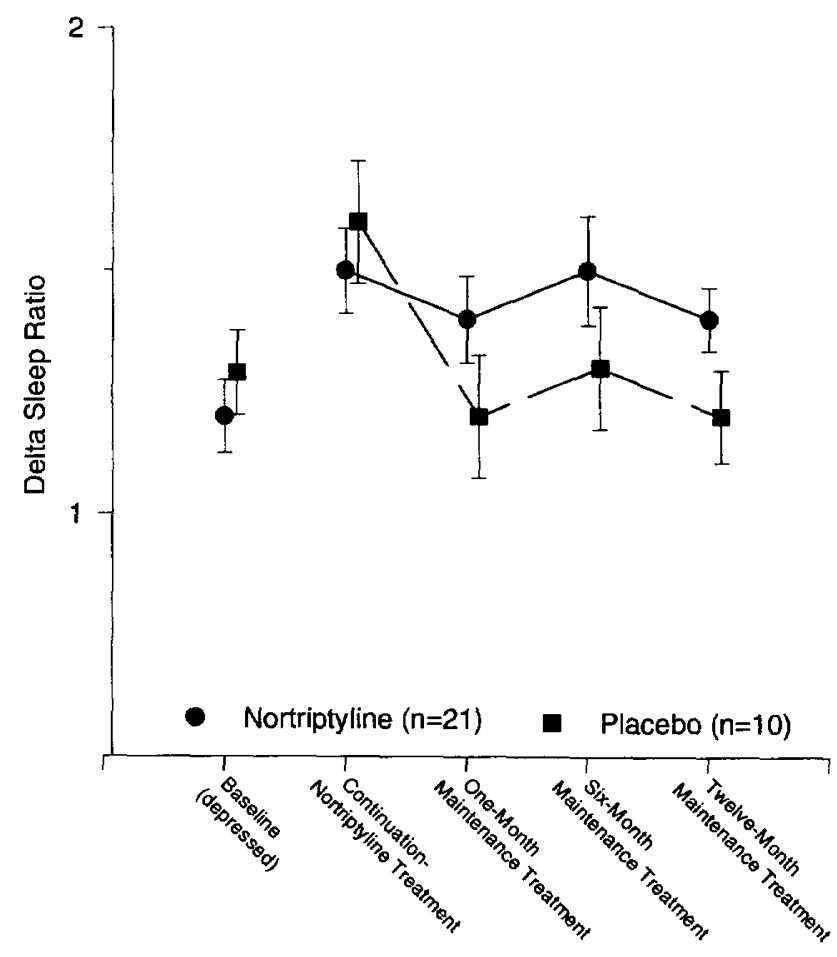

Figure 4. Nortriptyline increases delta EEG counts early in the night both acutely and persistently. Delta ratio is defined as the number of EEG delta counts per minute in the first NREM period divided by the number of EEG counts per minute in the second NREM period. A higher number represents the more "normal" physiological state, with maximal delta activity in the first NREM period. Nortriptyline and placebo-treated patients showed a trend toward a group difference $(F=3.24, p<.09)$. Nortriptyline $(\mathrm{n}=21) \square$ Placebo $(\mathrm{n}=10)$

predict outcome. We examined the change in REM latency from baseline $\left(T_{1}\right)$ to early nortriptyline treatment $\left(T_{2}\right)$. Patients who suffered a recurrence during the subsequent year had a greater REM latency than those who remained well $(t=2.20, p<.03)$.

In the next analysis we contrasted a group of patients who completed 1 year of maintenance treatment on placebo ( $n=14$ ) with those who had a recurrence during maintenance treatment with placebo $(n=25)$. At the baseline $\left(T_{1}\right)$ sleep evaluation we found no significant differences among completers versus recurrences on any of the sleep measures outlined in Table 1 . We repeated this analysis during early nortriptyline continuation $\left(T_{2}\right)$ found that placebo-treated patients who completed 1 year of maintenance therapy had a lower $T_{2}$ PSQI score, that is, better subjective sleep quality, than those who subsequently had a recurrence (3.9 \pm 2.2 vs. $7.2 \pm 4.2 ; \mathrm{t}=-3.05, p<.005$ ).

We completed a similar analysis contrasting nortriptyline-treated patients who completed 1 year of maintenance pharmacotherapy $(n=24)$ with those who suffered a recurrence $(n=9)$ during maintenance pharmacotherapy. Completers had significantly higher $T_{2}$ REM density than subjects having recurrences $(2.6 \pm 1.0$ vs. $2.0 \pm 0.5 ; t=2.21, p<.05)$ and significantly greater $T_{2}$ automated REM counts (945 \pm 470 vs. $635 \pm 225 ; t=$ $2.51, p<.02$ ) during early continuation therapy with nortriptyline. The groups did not differ for the other EEG sleep measures shown in Table 1.

Because PSQI scores were related to recurrence or time to recurrence in several of the previous analyses, we examined whether subjective sleep quality may reflect depression severity or personality style. Among the 69 patients randomized to nortriptyline or placebo, PSQI scores at $T_{2}$ correlated significantly with HRSD scores $(r=0.49, p<.0001)$, but not with either the total score or the eccentric, dramatic, or anxious subscales, or the Personality Assessment Form (Shea et al. 1987). However, when we entered Hamilton scores into the Cox proportional hazards model with $T_{2}$ sleep variables as above, HRSD scores were not significantly associated with time to recurrence.

\section{DISCUSSION}

The acute effects of nortriptyline on EEG sleep persist over the course of 1 year. Specifically, maintenance nortriptyline treatment decreases REM percent and increases REM density and shows a trend toward increasing delta EEG activity in the first NREM period. Nortriptyline has a mild salutary effect on sleep apnea and did not increase PLMs. Better subjective sleep quality and greater phasic REM activity during continuation treatment, as well as maintenance treatment with nortriptyline and IPT, all decreased the likelihood of recurrence over 1 year of maintenance treatment.

The results of this investigation largely, but not completely, parallel those reported by Kupfer et al. (1994) in a sample of midlife depressed patients treated with imipramine. In particular, neither found any significant effects of antidepressants on sleep continuity and relatively minor effects on sleep stage architecture. In contrast, REM sleep percent was diminished, REM phasic activity increased, and delta ratio increased both acutely and persistently. The similarities between our study and that of Kupfer et al. demonstrate some generalizability for different specific tricyclic agents across midlife and late-life depressed patient samples.

Our patients did not have clinically significant sleep apnea or PLMs. Nevertheless, nortriptyline decreased the number of apnea events, both acutely and during maintenance treatment. Tricyclic and SSRI antidepressants have previously been reported to decrease obstructive sleep apnea (Brownell et al. 1982; Hanzel et al. 1991). These studies have also found a beneficial effect 
of antidepressants during NREM apneas, suggesting that changes in muscle tone, rather than a reduction in REM sleep, are likely responsible for this effect.

Although antidepressant medications are often assumed to worsen PLMs (Ware et al. 1984), we did not find such an effect in our patients. In fact, there is little evidence of any change in PLMs during nortriptyline treatment. Alhtough it remains possible that patients with increased PLMs during tricyclic therapy would not respond to initial treatment and thereby not enter these types of analyses, our previous study on the acute effects of nortriptyline suggests that this is not the case (Reynolds et al. 1991).

Our main measure of the longitudinal effects of nortriptyline contrasted patients who remained well on nortriptyline and those who remained well while taking placebo during maintenance therapy. This type of comparison may have biased against finding more potent effects of nortriptyline on EEG sleep; the group of patients who remained well on placebo might be expected to have better sleep in general than other patients. Furthermore, they may represent a "special" group of survivors who do not require continued medication. However, the absence of significant differences in baseline sleep EEG measures between placebo completers and recurrences argues against this explanation.

Low phasic REM activity was associated with recurrence, particularly among patients maintained on nortriptyline. This finding is compatible with Vogel's "REM pressure" hypothesis (Vogel et al. 1980). Specifically, patients who benefit most from a treatment that suppresses the expression of REM sleep (e.g., REM deprivation, REM-suppressing drugs) are those who show the greatest "REM pressure." In this case REM pressure is expressed through phasic REM activity.

In contrast to earlier reports (Kupfer et al. 1990, 1993), we did not find that either total delta EEG activity or the temporal pattern of delta activity (reflected by delta ratio) were associated with recurrence of depression or time to recurrence. However, our patients have been followed for only 1 year, compared to the 3-year follow-up in Kupfer's study. This difference is important, because the median time to recurrence of the Kupfer subgroups was 40 to 100 weeks. Results of the current study, together with Kupfer's findings on recurrence and previous data suggesting an association between acute REM suppression and acute treatment response (Kupfer et al. 1981; Reynolds et al. 1991); raise the following hypothesis--that acute treatment response and early recurrence relate to tonic and phasic REM, but that longer-term recurrence relates more to EEG delta activity.

In neurobiological terms, both increased REM and reduced delta activity can result from increased cholinergic activation relative to monoaminergic and GABA ergic activity in brainstem nuclei and in thalamo-cortical projecting neurons (Steriade 1992). Augmentation of REM sleep and phasic REM activity appear early in episodes of depression (Kupfer et al. 1988), whereas delta activity and slow-wave sleep are more persistent characteristics. Thus, a reduced delta may represent an underlying vulnerability factor for depression resulting from relative cholinergic overactivity. With further heightening of cholinergic activation during an episode of depression, state-related REM changes may then appear. One study of cholinergic REM induction supports the hypothesis of greater cholinergic sensitivity during an episode of depression compared to recovery (Riemann and Berger 1989), although this finding has not been consistent (Sitaram et al. 1982). Longitudinal studies of cholinergic sensitivity using spectral analysis or periodamplitude measures of delta EEG activity would be more sensitive to subtle changes in slow-wave sleep.

A second potential explanation for the lack of association between delta EEG activity and recurrence in the present study is that delta EEG activity decreases dramatically with age among depressed patients (Reynolds et al. 1990). Age-related changes result from decreased delta-wave amplitude, rather than delta wave incidence (Feinberg et al. 1984), but our detection algorithm is sensitive to both aspects. Elderly depressed patients may have insufficient delta activity over $75 \mu \mathrm{V}$ for an association with recurrence to be seen. It is conceivable that higher-amplitude delta activity in particular is correlated with protection against recurrence.

Subjective sleep quality measured by the PSQI was also associated with recurrence. We have previously found that the PSQI changes significantly during acute nortriptyline treatment, although it does not correlate with acute changes in sleep EEG or HDRS scores (Reynolds et al. 1991). In the current study PSQI changes correlated significantly with depression ratings. However, depression ratings did not correlate with time to recurrence, suggesting an independent association between sleep quality and recurrence. Among healthy elderly subjects, "good" and "poor" sleep quality do not appear to relate to objective EEG sleep measures or the frequency of daytime napping (Buysse 1991). Thus, subjective sleep quality appears to reflect a unique dimension of subjective state, distinct from objective sleep measures, with implications for long-term functioning.

Finally, we found that maintenance treatment with nortriptyline and IPT were significantly associated with a decreased likelihood of recurrence. This finding confirms previous reports by Kupfer et al. (1990) and Frank et al. $(1990,1991)$ on the role of both medications and IPT in preventing recurrences. Our findings also support the utility of examining both biological and nonbiological treatment variables in depression outcome research. 


\section{ACKNOWLEDGMENT}

Supported in part by NIMH Grant \#MH52247, MH30915, MH43832, MH37869, MH00295, and MH48891.

\section{REFERENCES}

American Psychiatric Association (1993): American Psychiatric Association practice guideline for major depressive disorder in adults. Am J Psychiatr 150(4):April 1993 Supplement

Benca RM, Obermeyer WH, Thisted RA, Gillin JC (1992): Sleep and psychiatric disorders: A meta-analysis. Arch Gen Psychiatry 49:651-668

Brownell LG, West P, Sweatman P, Acres JC, Kryger MH (1982): Protriptyline in obstructive sleep apnea: A double-blind trial. N Engl J Med 307:1037-1042

Buysse DJ (1991): Drugs affecting sleep, sleepiness and performance. In Monk TH (ed), Sleep, Sleepiness and Performance. New York, Wiley, pp 249-306

Buysse DJ, Reynolds CF, Monk TH, Berman SR, Kupfer DJ (1989): The Pittsburgh Sleep Quality Index (PSQI): A new instrument for psychatric research and practice. Psychiatr Res 28:193-213

Doman J, Detka C, Hoffman T, Kesicki D, Monahan JP, Buysse DJ, Reynolds CF, Cobie PA, Matzzie J, Kupfer DJ (1995): Automating the sleep laboratory: Implementation and validation of digital recording and analysis. Int J Biomed Comput 38:277-290

Dunleavy DLF, Oswald I (1973): Phenelzine, mood response, and sleep. Arch Gen Psychiatr 28:353-356

Dunleavy DLF, Brezinova V, Oswald I, MacLean AW, Tinker M (1972): Changes during weeks in effects of tricyclic drugs on the human sleep brain. Br J Psychiatr 120:663672

Feinberg I, March JD, Floyd TC, Fein G, Aminoff MJ (1984): Delta $(.5-3 \mathrm{~Hz})$ EEG waveforms during sleep in young and elderly normal subjects. In Chase MH, Weitzman ED (eds), Sleep Disorders: Basic and Clinical Research. New York, Spectrum, pp 449-462

Frank E, Kupfer DJ, Perel JM, Cornes C, Jarrett DB, Mallinger AG, Thase ME, McEachran AB, Grochocinski VJ (1990): Three-year outcomes for maintenance therapies in recurrent depression. Arch Gen Psychiatr 47:1093-1099

Frank E, Kupfer DJ, Wagner EF, McEachran AB, Cornes C (1991): Efficacy of interpersonal psychotherapy as a maintenance treatment of recurrent depression: Contributing factors. Arch Gen Psychiatr 48:1053-1059

Giles DE, Jarrett RB, Roffwarg HP, Rush AJ (1987): Reduced rapid eye movement latency: A predictor of recurrence in depression. Neuropsychopharmacology 1:33-39

Hamilton M (1960): A rating scale for depression. J Neurol Neurosurg Psychiatr 12:56-62

Hanzel DA, Proia NG, Hudgel DW (1991): Response of obstructive sleep apnea to fluoxetine and protriptyline. Chest 100:416-421

Knowles JB, MacLean AW (1990): Age related changes in sleep in depressed and healthy subjects: A meta-analysis. Neuropsychopharmacology 3:251-259
Kupfer DJ, Spiker DG, Coble PA, Neil JF, Ulrich R, Shaw DH (1981): Sleep and treatment prediction in endogenous depression. Am J Psychiatr 138:429-434

Kupfer DJ, Ulrich RF, Coble PA (1984): Application of automated REM and slow-wave sleep analysis: II. Testing the assumptions of the two-process model of sleep regulation in normal and depressed subjects. Psychiatry Res $13: 335-343$

Kupfer DJ, Frank E, Grochocinski VJ, Gregor M, McEachran $A B$ (1988): Electroencephalographic sleep profiles in recurrent depression: A longitudinal investigation. Arch Gen Psychiatr 45:678-681

Kupfer DJ, Ehlers CL, Pollock BG, Nathan RS, Perel JM (1989): Clomipramine and EEG sleep in depression. Psychiatr Res 30:165-180

Kupfer DJ, Frank E, McEachran AB, Grochocinski VJ (1990): Delta sleep ratio: A biological correlate of early recurrence in unipolar affective disorder. Arch Gen Psychiatr 47:1100-1105

Kupfer DJ, Frank E, McEachran AB, Grochocinski VJ, Ehlers CL (1993): EEG sleep correlates of recurrence of depression on active medication. Depression 1:300-308

Kupfer DJ, Ehlers CL, Frank E, Grochocinski V, McEachran A, Buhari A (1994): Persistent effects of antidepressants: EEG sleep studies in depressed patients during maintenance treatment. Biol Psychiatr 35:781-793

Montgomery I, Oswald I, Morgan I, Adam K (1983): Trazodone enhances sleep in subjective quality but not in objective duration. Br J Clin Pharmacol 16:139-144

Mouret J, Lemoine P, Minuit MP, Benkelfat C, Renardet M (1988): Effects of trazodone on the sleep of depressed subjects-A polygraphic study. Psychopharmacology 95(Suppl):S37-S43

Reynolds CF, Kupfer DJ (1987): Sleep research in affective illness: State of the art circa 1987 (state-of-the-art review). Sleep 10:199-215

Reynolds CF, Kupfer DJ, Thase ME, Frank E, Jarrett DB, Coble PA, Hoch CC, Buysse DI, Simons AD, Houck PR (1990): Sleep, gender, and depression: An analysis of gender effects on the electroencephalographic sleep of 302 depressed outpatients. Biol Psychiatr 28:673-684

Reynolds CF, Hoch CC, Buysse DJ, George CJ, Houck PR, Mazumdar S, Miller M, Pollock BG, Rifai H, Frank E, Cornes C, Moryce RK, Kupfer DJ (1991): Sleep in latelife recurrent depression: Changes during early continuation therapy with nortriptyline. Neuropsychopharmacology 5:85-96

Reynolds CF, Frank E, Perel JM, Imber SD, Cornes C, Morycz RK, Mazumdar S, Miller MD, Pollock BG, Rifai AH, Stack JA, George CJ, Houck PR, Kupfer DJ (1992): Combined Pharmacotherapy in the Acute and Continuation Treatment of Elderly Patients with Recurrent Major Depression: A Preliminary Report. Am J Psychiatr 149(12): $1687-1692$

Riemann D, Berger M (1989): EEG sleep in depression and in remission and the REM sleep response to the cholinergic agonist RS 86. Neuropsychopharmacology 2:145-152

Shea MT, Glass D, Pilkonis PA, Watkins J, Docherty J (1987): Frequency and implications of personality disorders in a sample of depressed outpatients. J Personality Dis $1: 27-42$ 
Sitaram N, Nurnberger JI, Gershon ES, Gillin JC (1982): Cholinergic regulation of mood and REM sleep: Potential model and marker of vulnerability to affective disorders. Am J Psychiatr 139:571-576

Spitzer RL (1978): Schedule for Affective Disorders and Schizophrenia - Lifetime Version (SADS-L). New York, New York State Psychiatric Institute

Steriade M (1992): Basic mechanisms of sleep generation. Neurology 42:9-18
Vogel GW, Vogel F, McAbee RS, Thurmond AJ (1980): Improvement of depression by REM sleep deprivation. Arch Gen Psychiatr 37:247-253

Ware JC, Brown FW, Moorad PJ (1984): Nocturnal myoclonus and tricyclic antidepressants. Sleep Res 13:72

Ware JC, Brown FW, Moorad PJ, Pittard JT, Cobert B (1989): Effects on sleep: A double-blind study comparing trimipramine to imipramine in depressed insomniac patients. Sleep 12:537-549 